\title{
STABILITY RESULTS ON AGE-STRUCTURED SIS EPIDEMIC MODEL WITH COUPLING IMPULSIVE EFFECT
}

\author{
HELONG LIU, HOUBAO XU, JINGYUAN YU, AND GUANGTIAN ZHU
}

Received 29 June 2005; Accepted 10 October 2005

We develop an age-structured epidemic model for malaria with impulsive effect, and consider the effect of blood transfusion and infected-vector transmission. Transmission rates depend on age. We derive the condition in which eradication solution is locally asymptotically stable. The condition shows that large enough pulse reducing proportion and relatively small interpulse time lead to the eradication of the diseases.

Copyright (C) 2006 Helong Liu et al. This is an open access article distributed under the Creative Commons Attribution License, which permits unrestricted use, distribution, and reproduction in any medium, provided the original work is properly cited.

\section{Introduction}

Malaria is a common and serious disease. It is reported that the incidence of malaria in the world may be in the order of 300 millon clinical cases each year. Malaria mortality is estimated at almost 2 million deaths worldwide per year. The vast number of malaria deaths occur among young children in Africa, especially in remote rural areas. In addition, an estimated over 2 billion people are at risk of infection, no vaccines are available for the disease $[12,14,18]$.

Malaria in humans is due to infection by one of four Plasmodium ( $P$. falciparum, $P$. vivax, $P$. ovale and $P$. malariae). Malaria parasites are normally transmitted from one person to another via mosquitos of the Anopheles species. The form of the parasite that can infect mosquitos is called gametocyte. Gametocytes start developing in capillaries of the inner organs of infected persons after invasion of the blood by merozoites. Mature gametocytes, which are infective to mosquitos, appear in the peripheral blood some 3 to 10 days later. The female Anopheles mosquito ingests malaria gametocytes when it takes a blood meal from an infected person. The parasite then needs a period of development in the mosquito before it can infect other people again $[1,3,17]$.

Malaria infected persons who donate blood before the onset of clinical symptoms, but after merozoites have entered the blood stream from the liver, can unknowingly transmit

Hindawi Publishing Corporation

Discrete Dynamics in Nature and Society

Volume 2006, Article ID 83489, Pages 1-11

DOI 10.1155/DDNS/2006/83489 
malaria through their blood donation. Similarly, malaria may be transmitted by contaminated needles and syringes. So, individuals in incubation period can also infect susceptible population.

An effective way to prevent the malaria epidemic is to control mosquitoes, which has been one of the major intensive efforts in many years. Constantly massive spraying of insecticides or eliminating breeding sites has greatly limited malaria in some area. However, because economic condition, applying these strategies are difficult in many developing countries.

Scholars have recognized the age structure of a population affects the dynamics of disease transmission, so various age-structured epidemic models have been investigated $[6,7,9]$. However, the dynamics of ecological communities require attention to the interplay between population dynamics and evolutionary change in interacting species [11]. In the systems, fitness is not constant, and may vary with species densities. So, it is necessary and also it is of practical significance to consider the dynamic behavior about the vector-host model. Some authors have studied the vector-host models. In [10], a vectorhost model for the spread of Chagas disease has been studied. In [5], the models couple host evolution with parasite population, and include the relevant genetic structure of malaria.

Scholars mentioned above used continuous equations to study the epidemiological dynamic models, while ignoring the external disturbance. However, in the natural world, the optimization and control of some biological phenomena are impulse. In this dissertation, instead of constantly spraying of insecticides, we establish epidemiological model with impulsive effect. In order to reflect the age structure of a population affects the dynamics of disease transmission, we incorporate age into the model, and assume that the infection rate depend on the age.

This paper is organized as follows: Section 2 introduces vector-host model with impulsive effect. In Section 3 we establish the existence and uniqueness of solution for the model equations. In Section 4, we get the condition of the local asymptotic stability of the infection-free solution.

\section{The model formulation}

In this section, we assume that the host population is in a stationary demographic state, whose total size is constant $N$. Let $N(a), 0 \leq a \leq r_{m}$ ( $r_{m}$ denotes the highest age attained by the individuals in the host population) be the age density of the total number of individuals, and $N(a)$ satisfies

$$
N(a)=\mu^{*} N e^{-\int_{0}^{a} \mu_{1}(s) d s}
$$

$\mu_{1}(a)$ is the instantaneous death rate at age $a$ of the host population, $\mu^{*}$ is the crude death rate, we assume that $\mu(a)$ is nonnegative, locally integrable on $\left[0, r_{m}\right)$, and satisfies

$$
\int_{0}^{r_{m}} \mu(a) d a=\infty
$$


$\mu^{*}$ satisfies

$$
\mu^{*} \int_{0}^{r_{m}} f(a) d a=1
$$

where $f(a)=e^{-\int_{0}^{a} \mu(s) d s}$ is the survival function. We can get the relation $N(a)=\mu^{*} N f(a)$. The host population is divided into two groups: susceptible $S(a, t)$ (who are healthy but can be infected), infected $I(a, t)$ (which includes latent individuals, since individuals in incubation period can also infect susceptible population), $S(a, t), I(a, t)$ is the age-densities of respectively the susceptible and infected population at time $t . N(a)$ also satisfies

$$
N(a)=S(a, t)+I(a, t) .
$$

Let $M(t)$ denote the number of susceptible vectors (mosquito populations) at time $t$, $P(t)$ the number of infected vectors at time $t . b, \mu_{2}$ is the birth and death rate of vectors, respectively. Since blood transfusion, or using contaminated needles and syringes, susceptibles $S(a, t)$ can be infected, and become infected individuals at a transmission $\beta_{1}(a)$. Susceptibles $S(a, t)$ are infected by infected vector, and go into infected class at a transmission rate $\beta_{2}$. The number of new of vectors by infected hosts depend on the transmission rate $\gamma(a)$. The infected population can recover, and go into susceptible population at a transmission rate $\alpha$. In order to control the size of mosquito, we apply the pulse spraying strategy of insecticides. We spray insecticides upon mosquito at time $n T$ every $T$ months, $T$ is the period of spraying, $n T$ is the time at which we apply the $n$th $\left(n \in N_{+}\right)$pulse, and $n T^{-}$is the time just before applying the $n$th pulse. Every pulse can reduce a fraction $p$ of mosquito population. We obtain the following system of equations that describe the dynamics of the model:

$$
\begin{gathered}
\frac{\partial S}{\partial t}+\frac{\partial S}{\partial a}=-\left(\mu_{1}(a)+\int_{0}^{r_{m}} \beta_{1}(a) I(a, t) d a+\beta_{2} P(t)\right) S(a, t)+\alpha I, \quad 0<a<r_{m}, t \neq n T, n \in N_{+}, \\
S(a, n T)=S\left(a, n T^{-}\right), \quad t=n T, \\
\frac{\partial I}{\partial t}+\frac{\partial I}{\partial a}=\left(\int_{0}^{r_{m}} \beta_{1}(a) I(a, t) d a+\beta_{2} P(t)\right) S(a, t)-\left(\mu_{1}(a)+\alpha\right) I, \quad 0<a<r_{m}, t \neq n T, \\
I(a, n T)=I\left(a, n T^{-}\right), \quad 0 \leq a<r_{m}, \\
\frac{d M}{d t}=b-M \int_{0}^{r_{m}} \gamma(a) I(a, t) d a-\mu_{2} M, \quad t \neq n T, \\
M(n T)=(1-p) M\left(n T^{-}\right), \quad t=n T, \\
\frac{d P}{d t}=M \int_{0}^{r_{m}} \gamma(a) I(a, t) d a-\mu_{2} P, \quad t \neq n T, \\
P(n T)=(1-p) P\left(n T^{-}\right), \quad t=n T,
\end{gathered}
$$


4 Stability results on age-structured SIS epidemic model

with boundary conditions:

$$
S(0, t)=\mu^{*} N, \quad I(0, t)=0,
$$

and initial conditions:

$$
S(0)=S_{0}(a) \geq 0, \quad I(a, 0)=I_{0}(a) \geq 0, \quad M(0)=M_{0} \geq 0, \quad P(0)=P_{0} \geq 0,
$$

where $S_{0}(a), I_{0}(a) \in L\left(0, r_{m}\right)$.

Let $s(a, t)=S(a, t) / N(a), i(a, t)=I(a, t) / N(a)$. The system (2.5) can be written in a simple form (we still let $S(a, t), I(a, t)$ denote the age-densities of the susceptible and infected population, resp.):

$$
\begin{gathered}
\frac{\partial S}{\partial t}+\frac{\partial S}{\partial a}=-\left(\int_{0}^{r_{m}} \beta_{1}(a) N(a) I(a, t) d a+\beta_{2} P(t)\right) S(a, t)+\alpha I, \quad 0<a<r_{m}, t \neq n T, n \in N_{+}, \\
S(a, n T)=S\left(a, n T^{-}\right), \quad t=n T, \\
\frac{\partial I}{\partial t}+\frac{\partial I}{\partial a}=\left(\int_{0}^{r_{m}} \beta_{1}(a) N(a) I(a, t) d a+\beta_{2} P(t)\right) S(a, t)-\alpha I, \quad 0<a<r_{m}, t \neq n T, \\
I(a, n T)=I\left(a, n T^{-}\right), \quad 0 \leq a<r_{m}, \\
\frac{d M}{d t}=b-M \int_{0}^{r_{m}} \gamma(a) N(a) I(a, t) d a-\mu_{2} M, \quad t \neq n T, \\
M(n T)=(1-p) M\left(n T^{-}\right), \quad t=n T, \\
\frac{d P}{d t}=M \int_{0}^{r_{m}} \gamma(a) N(a) I(a, t) d a-\mu_{2} P, \quad t \neq n T, \\
P(n T)=(1-p) P\left(n T^{-}\right), \quad t=n T,
\end{gathered}
$$

with boundary conditions:

$$
S(0, t)=1, \quad I(0, t)=0,
$$

and initial conditions:

$$
S(a, 0)=S_{0}(a) \geq 0, \quad I(a, 0)=I_{0}(a) \geq 0, \quad M(0)=M_{0} \geq 0, \quad P(0)=P_{0} \geq 0 .
$$

\section{Existence and uniqueness of solution}

Since $S(a, t)+I(a, t)=1$, it is sufficient to consider the system (2.8) in terms only $I(a, t)$, $M(t), P(t)$, once these functions are known, $S(a, t)$ can be obtain by $S(a, t)=1-I(a, t)$. 
Then the system (2.8) is written to a simpler form:

$$
\begin{gathered}
\frac{\partial I}{\partial t}+\frac{\partial I}{\partial a}=\left(\int_{0}^{r_{m}} \beta_{1}(a) N(a) I(a, t) d a+\beta_{2} P(t)\right)(1-I(a, t))-\alpha I, \quad 0<a<r_{m}, t \neq n T, \\
I(a, n T)=I\left(a, n T^{-}\right), \quad 0 \leq a<r_{m}, \\
\frac{d M}{d t}=b-M \int_{0}^{r_{m}} \gamma(a) N(a) I(a, t) d a-\mu_{2} M, \quad t \neq n T, \\
M(n T)=(1-p) M\left(n T^{-}\right), \quad t=n T, \\
\frac{d P}{d t}=M \int_{0}^{r_{m}} \gamma(a) N(a) I(a, t) d a-\mu_{2} P, \quad t \neq n T, \\
P(n T)=(1-p) P\left(n T^{-}\right), \quad t=n T,
\end{gathered}
$$

with boundary conditions: $I(0, t)=0$, and initial conditions:

$$
I(a, 0)=I_{0}(a) \geq 0, \quad M(0)=M_{0} \geq 0, \quad P(0)=P_{0} \geq 0 .
$$

We note that the system

$$
\begin{gathered}
\frac{\partial I}{\partial t}+\frac{\partial I}{\partial a}=\left(\int_{0}^{r_{m}} \beta_{1}(a) N(a) I(a, t) d a+\beta_{2} P(t)\right)(1-I(a, t))-\alpha I, \quad 0<a<r_{m}, 0<t<\infty, \\
\frac{d M}{d t}=b-M \int_{0}^{r_{m}} \gamma(a) N(a) I(a, t) d a-\mu_{2} M, \quad 0<t<\infty, \\
\frac{d P}{d t}=M \int_{0}^{r_{m}} \gamma(a) N(a) I(a, t) d a-\mu_{2} P, \quad 0<t<\infty,
\end{gathered}
$$

with boundary conditions: $I(0, t)=0$, and initial conditions:

$$
I(a, 0)=I_{0}(a) \geq 0, \quad M(0)=M_{0} \geq 0, \quad P(0)=P_{0} \geq 0,
$$

exists a unique nonnegative solution (applying a semigroup setting to seek the solution in a weak sense $[13,16]$, here we omit the proof). Let $\left(I_{0}(a, t), M_{0}(t), P_{0}(t)\right), 0 \leq a<r_{m}$, $t \in[0, \infty)$ denote the solution of the system (3.3).

For $n \in N_{+}$, the system:

$$
\begin{gathered}
\frac{\partial I_{n}}{\partial t}+\frac{\partial I_{n}}{\partial a}=\left(\int_{0}^{r_{m}} \beta_{1}(a) N(a) I_{n}(a, t) d a+\beta_{2} P_{n}(t)\right)\left(1-I_{n}(a, t)\right)-\alpha I_{n}, \quad 0<a<r_{m}, n T<t<\infty, \\
\frac{d M_{n}}{d t}=b-M_{n} \int_{0}^{r_{m}} \gamma(a) N(a) I_{n}(a, t) d a-\mu_{2} M_{n}, \quad n T<t<\infty, \\
\frac{d P_{n}}{d t}=M_{n} \int_{0}^{r_{m}} \gamma(a) N(a) I_{n}(a, t) d a-\mu_{2} P_{n}, \quad n T<t<\infty,
\end{gathered}
$$


6 Stability results on age-structured SIS epidemic model

with boundary conditions: $I_{n}(0, t)=0$, and initial conditions:

$$
I_{n}(a, n T)=I_{n-1}\left(a, n T^{-}\right), \quad M_{n}(n T)=M_{n-1}\left(n T^{-}\right), \quad P_{n}(n T)=P_{n-1}\left(n T^{-}\right),
$$

is equal to the system

$$
\begin{gathered}
\frac{\partial \bar{I}_{n}}{\partial \tau}+\frac{\partial \bar{I}_{n}}{\partial a}=\left(\int_{0}^{r_{m}} \beta_{1}(a) N(a) \bar{I}_{n}(a, \tau) d a+\beta_{2} \bar{P}_{n}(\tau)\right)\left(1-\bar{I}_{n}(a, \tau)\right)-\alpha \bar{I}_{n}, \quad 0<a<r_{m}, 0<\tau<\infty \\
\frac{d \bar{M}_{n}}{d \tau}=b-\bar{M}_{n} \int_{0}^{r_{m}} \gamma(a) N(a) \bar{I}_{n}(a, \tau) d a-\mu_{2} \bar{M}_{n}, \quad 0<\tau<\infty, \\
\frac{d \bar{P}_{n}}{d \tau}=\bar{M}_{n} \int_{0}^{r_{m}} \gamma(a) N(a) \bar{I}_{n}(a, \tau) d a-\mu_{2} \bar{P}_{n}, \quad 0<\tau<\infty
\end{gathered}
$$

with boundary: $\bar{I}_{n}(0, \tau)=0$, and initial conditions:

$$
\bar{I}_{n}(a, 0)=I_{n-1}\left(a, n T^{-}\right), \quad \bar{M}_{n}(0)=M_{n-1}\left(n T^{-}\right), \quad \bar{P}_{n}(n T)=P_{n-1}\left(n T^{-}\right),
$$

where $\tau=t-n T, \bar{I}_{n}(a, \tau)=I_{n}(a, \tau+n T), \bar{M}_{n}(\tau)=M_{n}(\tau+n T), \bar{P}_{n}=P_{n}(\tau+n T), n \in N_{+}$.

So we obtain that the system (3.5) exist an unique nonnegative solution, denote it by $\left(I_{n}(a, t), M_{n}(t), P_{n}(t)\right), 0 \leq a<r_{m}, n T \leq t<\infty, n \in N_{+}$.

Let $(I(a, t), M(t), P(t))$ denote the solution of the system (3.1), we have the representation:

$$
(I(a, t), M(t), P(t))=\left(I_{n}(a, t), M_{n}(t), P_{n}(t)\right), \quad 0 \leq a<r_{m}, t \in[n T,(n+1) T), n \in N .
$$

From (3.9) and the system (3.3), (3.5), we can arrive at following result.

Theorem 3.1. If $\beta_{1}(a), \gamma(a) \in L^{\infty}\left(0, r_{m}\right)$, then the system (3.1) has a unique nonnegative solution $(I(a, t), M(t), P(t))$ with respect to initial data $\left(I_{0}(a), M_{0}, P_{0}\right) \in \Omega_{0}$, and $I(a, t), M(t)$, $P(t)$ are continuously differentiable on $a \in\left(0, r_{m}\right), t \in(n T,(n+1) t), n=0,1,2, \ldots$, where $\Omega_{0}=\left\{\left(I_{0}(a), M_{0}, P_{0}\right), M_{0}, P_{0} \in R_{+}, I_{0}(a) \in L\left(0, r_{m}\right), 0 \leq I_{0}(a)<1, I_{0}(0)=0\right\}$.

\section{Stability analysis}

The system (2.8) has a periodic infection-free solution $E^{*}($ see $[4,19])$

$$
\begin{gathered}
E^{*}=\left(1,0, M^{*}, 0\right), \\
M^{*}=\frac{b}{\mu_{2}}\left(1-\frac{p e^{\mu_{2}((n+1) T-t)}}{e^{\mu_{2} T}+p-1}\right), \quad t \in[n T,(n+1) T), n \in N,
\end{gathered}
$$

that is, the system (3.1) has a periodic infection-free solution $\left(0, M^{*}(t), 0\right)$.

In order to investigate the stability of the periodic infection-free solution $\left(0, M^{*}(t), 0\right)$ for the system (3.1), we first rewrite (3.1) into equations for small perturbations. Let

$$
I(a, t)=u(a, t), \quad M(t)=M^{*}(t)+v(t), \quad P(t)=\omega(t) .
$$


From (3.1), we have

$$
\begin{gathered}
\frac{\partial u}{\partial t}+\frac{\partial u}{\partial a}=\left(\int_{0}^{r_{m}} \beta_{1}(a) N(a) u(a, t) d a+\beta_{2} \omega(t)\right)(1-u(a, t))-\alpha u, \quad 0<a<r_{m}, t \neq n T, \\
u(a, n T)=u\left(a, n T^{-}\right), \quad 0 \leq a<r_{m}, t=n T, \\
\frac{d v}{d t}=-\left(M^{*}(t)+v\right) \int_{0}^{r_{m}} \gamma(a) N(a) u(a, t) d a-\mu_{2} v, \quad t \neq n T, \\
v(n T)=(1-p) v\left(n T^{-}\right), \quad t=n T, \\
\frac{d \omega}{d t}=\left(M^{*}(t)+v\right) \int_{0}^{r_{m}} \gamma(a) N(a) u(a, t) d a-\mu_{2} \omega, \quad t \neq n T, \\
\omega(n T)=(1-p) \omega\left(n T^{-}\right), \quad t=n T .
\end{gathered}
$$

It is easy to see that linearization of the system $(4.3)$ at $(0,0,0)$ leads to the following system:

$$
\begin{gathered}
\frac{\partial u}{\partial t}+\frac{\partial u}{\partial a}=\int_{0}^{r_{m}} \beta_{1}(a) N(a) u(a, t) d a+\beta_{2} \omega(t)-\alpha u, \quad 0<a<r_{m}, t \neq n T, \\
u(a, n T)=u\left(a, n T^{-}\right), \quad 0 \leq a<r_{m}, t=n T, \\
\frac{d \omega}{d t}=M^{*}(t) \int_{0}^{r_{m}} \gamma(a) N(a) u(a, t) d a-\mu_{2} \omega, \quad t \neq n T, \\
\omega(n T)=(1-p) \omega\left(n T^{-}\right), \quad t=n T,
\end{gathered}
$$

where we omit the equation for $v(t)$, since is determined from $u(a, t)$. We can obtain that the system (4.4) has a nonnegative solution for initial conditions $u(a, 0)=u_{0}(a) \geq 0$, $\omega(0)=\omega_{0} \geq 0$.

Now we consider a comparable system of the system (4.4):

$$
\begin{aligned}
\frac{\partial \bar{u}}{\partial t}+\frac{\partial \bar{u}}{\partial a} & =\int_{0}^{r_{m}} \beta_{1}(a) N(a) \bar{u}(a, t) d a+\beta_{2} \bar{\omega}(t)-\alpha \bar{u}, \quad 0<a<r_{m}, 0<t<\infty, \\
\frac{d \bar{\omega}}{d t} & =\frac{b}{\mu_{2}} \frac{e^{\mu_{2} T}-1}{e^{\mu_{2} T}+p-1} \int_{0}^{r_{m}} \gamma(a) N(a) \bar{u}(a, t) d a-\mu_{2} \bar{\omega}, \quad 0<t<\infty,
\end{aligned}
$$

with boundary condition $\bar{u}(0, t)=0$, and initial conditions $\bar{u}(a, 0)=u_{0}(a), \bar{\omega}(0)=\omega_{0}$. The system (4.5) has a unique nonnegative solution $(\bar{u}(a, t), \bar{\omega}(t))$, and

$$
0 \leq u(a, t) \leq \bar{u}(a, t), \quad 0 \leq \omega(t) \leq \bar{\omega}(t) .
$$

So, if we prove that the system $(4.5)$ is stable at $(0,0)$, then the system (4.4) is stable at $(0,0)$. 
8 Stability results on age-structured SIS epidemic model

We rewrite the system (4.5) in the following form:

$$
\frac{d \phi}{d t}=A \phi(t)+B \phi(t), \quad \phi(t)=\left(\phi_{1}(t), \phi_{2}(t)\right)^{T}, \quad \phi(0)=\phi_{0},
$$

where

$$
\begin{gathered}
A: D(A) \longrightarrow X, \quad X=L\left(0, r_{m}\right) \times R, D(A)=\left\{\left(\phi_{1}(a), \phi_{2}\right)^{T}, \phi_{1} \in W_{1}^{1}\left(0, r_{m}\right), \phi_{2} \in R\right\} \\
A=\left(\begin{array}{c}
-\frac{d \phi_{1}}{d a}-\alpha \phi_{1} \\
-\mu_{2} \phi_{2}
\end{array}\right), \\
B: X \longrightarrow X, \\
B=\left(\begin{array}{c}
\int_{0}^{r_{m}} \beta_{1}(a) N(a) \phi_{1}(a) d a+\beta_{2} \phi_{2} \\
\frac{b}{\mu_{2}} \frac{e^{\mu_{2} T}-1}{e^{\mu_{2} T}+p-1} \int_{0}^{r_{m}} \gamma(a) N(a) \phi_{1}(a) d a
\end{array}\right) .
\end{gathered}
$$

Clearly, $B$ is a bounded perturbation of $A$. Moreover, $B$ is compact. Next, we consider the eigenvalue problem for the linear system (4.7):

$$
\begin{aligned}
\frac{d \phi_{1}}{d a}+(\lambda+\alpha) \phi_{1} & =P_{1}\left(\phi_{1}\right)+\beta_{2} \phi_{2}, \\
\left(\lambda+\mu_{2}\right) \phi_{2} & =P_{2}\left(\phi_{1}\right),
\end{aligned}
$$

where

$$
\begin{aligned}
& P_{1}\left(\phi_{1}\right)=\int_{0}^{r_{m}} \beta_{1}(a) N(a) \phi_{1}(a) d a, \\
& P_{2}\left(\phi_{1}\right)=\frac{b}{\mu_{2}} \frac{e^{\mu_{2} T}-1}{e^{\mu_{2} T}+p-1} \int_{0}^{r_{m}} \gamma(a) N(a) \phi_{1}(a) d a .
\end{aligned}
$$

From (4.11), we obtain the characteristic equation of the system (4.7):

$$
\begin{gathered}
\frac{\beta_{2}}{\lambda+\mu_{2}} \frac{b}{\mu_{2}} \frac{e^{\mu_{2} T}-1}{e^{\mu_{2} T}+p-1} \int_{0}^{r_{m}} \gamma(a) N(a) \int_{0}^{a} e^{-(\lambda+\alpha)(a-s)} d s d a \\
+\int_{0}^{r_{m}} \beta_{1}(a) N(a) \int_{0}^{a} e^{-(\lambda+\alpha)(a-s)} d s d a=1 .
\end{gathered}
$$

We denote the left-hand side in (4.11) by $\Phi(\lambda)$. Let $\mu=\min \left\{\mu_{2}, \alpha\right\}$. If $\lambda \geq-\mu$ is real, then $\Phi(\lambda)$ is a decreasing function of $\lambda$, and $\lim _{\lambda \rightarrow+\infty} \Phi(\lambda)=0$. In addition, for any $\lambda$ with $\operatorname{Re} \lambda \geq 0$, we have $|\Phi(\lambda)| \leq \Phi(\operatorname{Re} \lambda)$. Let

$$
R_{0}=\frac{\beta_{2}}{\mu_{2}} \frac{b}{\mu_{2}} \frac{e^{\mu_{2} T}-1}{e^{\mu_{2} T}+p-1} \int_{0}^{r_{m}} \gamma(a) N(a) \int_{0}^{a} e^{-\alpha(a-s)} d s d a+\int_{0}^{r_{m}} \beta_{1}(a) N(a) \int_{0}^{a} e^{-\alpha(a-s)} d s d a .
$$


If $R_{0}<1$ and assume that $\lambda$ is a solution to (4.11) with $\operatorname{Re} \lambda \geq 0$, we have

$$
1=|\Phi(\lambda)| \leq \Phi(\operatorname{Re} \lambda) \leq \Phi(0)=R_{0}<1,
$$

which is a contradiction stemming from the assumption. So, we obtain that the solutions of (4.11) has negative real part. We get Proposition 4.1.

Proposition 4.1. If $R_{0}<1$, the eigenvalues of $A+B$ have negative real part.

It is easy to get Proposition 4.2.

Proposition 4.2. The operator $A$ is a closed linear operator and satisfies

$$
\left\|(\lambda-A)^{-n}\right\| \leq \frac{1}{(\lambda+\mu)^{n}}
$$

for $\lambda>-\mu$ and all positive integers $n$.

To prove that the system $(4.7)$ is stable at $(0,0)$, we need the following definition and lemma [2].

Definition 4.3. $C_{0}$-semigroup $T(t)$ is called quasi-compact if $T(t)=S(t)+G(t)$ with operator families $S(t)$ and $G(t)$ for $t \geq 0$ such that $\lim _{t \rightarrow \infty}\|S(t)\|=0$, and $G(t)$ is eventually compact.

Lemma 4.4. Let $H(t)$ be a quasi-compact $C_{0}$-semigroup and $E$ its infinitesimal generator. Then $\lim _{t \rightarrow \infty} e^{\epsilon t}\|H(t)\|=0$ for some $\epsilon>0$ if and only if all eigenvalues of $E$ have strictly negative real part.

Now we prove a lemma.

Lemma 4.5. Let $T(t)$ be a $C_{0}$-semigroup and $A+B$ its infinitesimal generator. If $R_{0}<1$, then the following inequality holds:

$$
\omega_{0}(A+B)<-\mu
$$

where $\omega_{0}(A+B)$ denotes the growth of the semigroup $T(t), t \geq 0$.

Proof. Since $A$ is a densely defined operator, from Proposition 4.2, we have that $A$ generates a $C_{0}$-semigroup on $X$, denote it by $S(t)$. From Proposition 4.2 , we have $\|S(t)\| \leq e^{-\mu t}$. Since $B$ is a bounded perturbation, $A+B$ also generates a $C_{0}$-semigroup in $X, T(t)$. Moreover, $B S(t): X \rightarrow X$ is compact for every $t>0$. From [15, Theorem 3], we get that $T(t)$ is quasi-compact. By Proposition 4.1 and Lemma 4.4 we have $\lim _{t \rightarrow \infty} e^{\mu t}\|T(t)\|=0$. This implies that the growth of $A+B$ does not exceed $-\mu$.

The lemma is completed.

If $\omega(A+B)<0$, the equilibrium $\phi=0$ of the system (4.11) is locally exponentially asymptotically stable in the sense that there exist $\epsilon>0, M \geq 1$ and $\gamma<0$ such that if $\phi_{0} \in X$ and $\left\|\phi_{0}\right\| \leq \epsilon$, then the solution $\phi\left(t, \phi_{0}\right)$ exists globally and $\left\|\phi\left(t, \phi_{0}\right)\right\| \leq \mathrm{Me}^{\gamma t}\left\|\phi_{0}\right\|$ for all $t \geq 0$ [16]. This implies that the equilibrium $\phi=0$ of the system (4.5) is locally asymptotically stable. 
From the above argument, and (4.6), we obtain that the system (4.4) is locally exponentially asymptotically stable at $(0,0)$. So we arrive at the following result.

Theorem 4.6. If $\beta_{1}(a), \gamma(a) \in L^{\infty}\left(0, r_{m}\right), \beta_{2} \geq 0$ and $R_{0}<1$, then the system (3.1), that is, the system (2.8), is locally exponentially asymptotically stable at $\left(0, M^{*}(t), 0\right)$.

From Theorem 4.6, we know the stability of the infection-free $E^{*}$ depends on $p$ and $T$. If $\beta_{1}(a)$ is small enough, and pulse reducing proportion of mosquito is increased and interpulse period is decreased such that $R_{0}<1$ holds. Consequently, the diseases is eradicated from the population. The results of this theoretical study are instructive to the study of other diseases, such as Chagas disease.

Here $R_{0}$ is not basic reproduction ratio [8]. Since the model is impulsive system, it is difficult to estimate basic reproduction ratio.

\section{References}

[1] R. M. Anderson and R. M. May, Infectious Diseases of Humans: Dynamics and Control, Oxford University Press, Oxford, UK, 1991.

[2] W. Arendt, A. Grabosch, G. Greiner, U. Groh, H. P. Lotz, U. Moustakas, R. Nagel, F. Neubrander, and U. Schlotterbeck, One-Parameter Semigroups of Positive Operators, Lecture Notes in Mathematics, vol. 1184, Springer, Berlin, 1986.

[3] T. Bradley, Malaria and drug resistance, MBChB special study module project report, Department of Microbiology and Immunology, University of Leicester, 1996, http://www-micro. msb.le.ac.uk/224/Bradley/Bradley.html.

[4] A. d'Onofrio, Stability properties of pulse vaccination strategy in SEIR epidemic model, Mathematical Biosciences 179 (2002), no. 1, 57-72.

[5] Z. Feng, D. L. Smith, F. E. McKenzie, and S. A. Levin, Coupling ecology and evolution: malaria and the S-gene across time scales, Mathematical Biosciences 189 (2004), no. 1, 1-19.

[6] D. Greenhalgh, Analytical threshold and stability results on age-structured epidemic models with vaccination, Theoretical Population Biology 33 (1988), no. 3, 266-290.

[7] __ Threshold and stability results for an epidemic model with an age-structured meeting rate, IMA: Journal of Mathematics Applied in Medicine and Biology 5 (1988), no. 2, 81-100.

[8] H. W. Hethcote, The mathematics of infectious diseases, SIAM Review 42 (2000), no. 4, 599-653.

[9] H. Inaba, Threshold and stability results for an age-structured epidemic model, Journal of Mathematical Biology 28 (1990), no. 4, 411-434.

[10] H. Inaba and H. Sekine, A mathematical model for Chagas disease with infection-age-dependent infectivity, Mathematical Biosciences 190 (2004), no. 1, 39-69.

[11] S. A. Levin and J. D. Udovic, A mathematical model of coevolving populations, The American Naturalist 111 (1977), no. 980, 657-675.

[12] Malaria Foundation International, Malaria: background information, http://www.malaria.org/ backgroundinfo.html, 1998.

[13] M. Martcheva and H. R. Thieme, Progression age enhanced backward bifurcation in an epidemic model with super-infection, Journal of Mathematical Biology 46 (2003), no. 5, 385-424.

[14] J. H. Pollard, Mathematical Models for the Growth of Human Population, Cambridge University Press, London, 1973.

[15] H. R. Thieme, Quasi-compact semigroups via bounded perturbation, Advances in Mathematical Population Dynamics-Molecules, Cells and Man (Houston, TX, 1995) (O. Arino, D. E. Axelrod, and M. Kimmel, eds.), Ser. Math. Biol. Med., vol. 6, World Scientific, New Jersey, 1997, pp. 691-711. 
[16] G. F. Webb, Theory of Nonlinear Age-Dependent Population Dynamics, Monographs and Textbooks in Pure and Applied Mathematics, vol. 89, Marcel Dekker, New York, 1985.

[17] W. H. Wernsdorfer, The importance of malaria in the world, Malaria. Volume 1: Epidemiology, Chemotherapy, Morphology, and Metabolism (J. P. Kreier, ed.), Academic Press, New York, 1980, pp. 1-93.

[18] WHO, Malaria, fact sheets, http://www.who.int/inf-fs/en/fact094.html, 1998.

[19] Y. Zhou and H. Liu, Stability of periodic solutions for an SIS model with pulse vaccination, Mathematical and Computer Modelling 38 (2003), no. 3-4, 299-308.

Helong Liu: Department of Mathematics, Xinyang Teachers College, Xinyang, Henan 464000, China E-mail address: liuhelong2004@yahoo.com.cn

Houbao Xu: Department of Mathematics, Beijing Institute of Technology, Beijing 100081, China E-mail address: xuhoubao@yahoo.com.cn

Jingyuan Yu: Beijing Institute of Information and Control Beijing 100037, China

E-mail address: yujingyuan@biic.net

Guangtian Zhu: Academy of Mathematics and System Science, C.A.S. Beijing 100080, China E-mail address: gtzhu@amss.ac.cn 


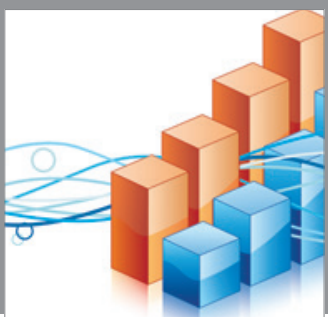

Advances in

Operations Research

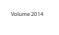

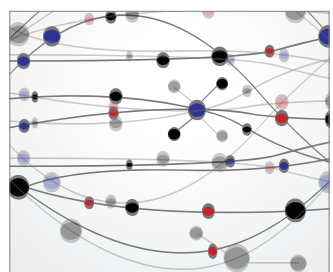

\section{The Scientific} World Journal
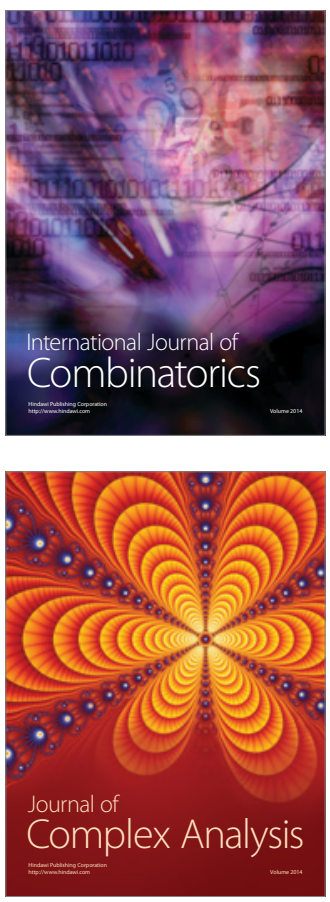

International Journal of

Mathematics and

Mathematical

Sciences
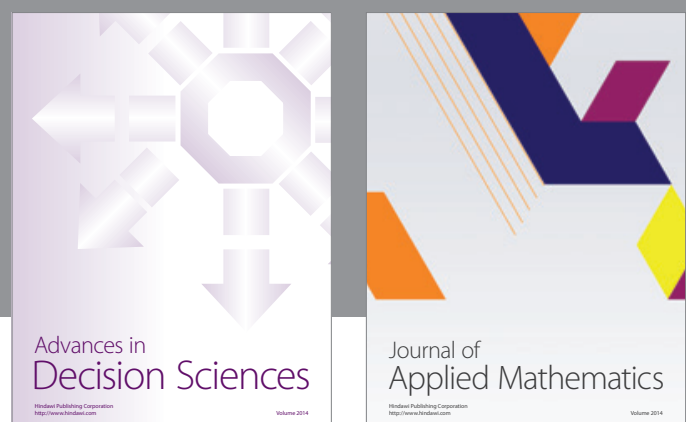

Journal of

Applied Mathematics
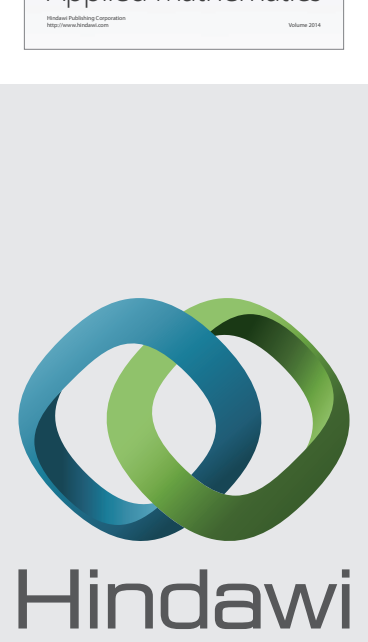

Submit your manuscripts at http://www.hindawi.com
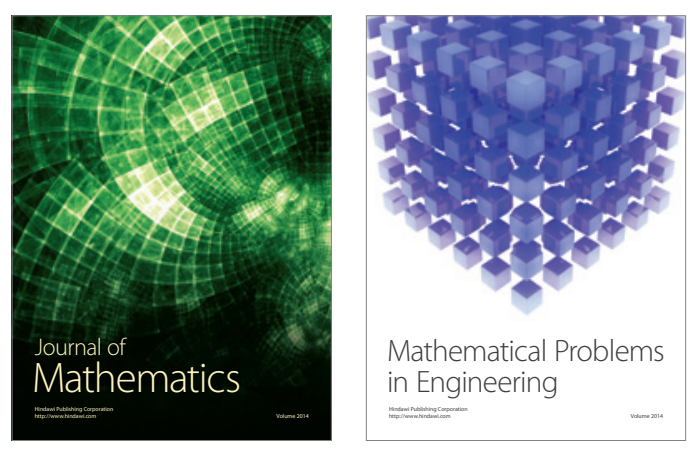

Mathematical Problems in Engineering


Journal of

Function Spaces
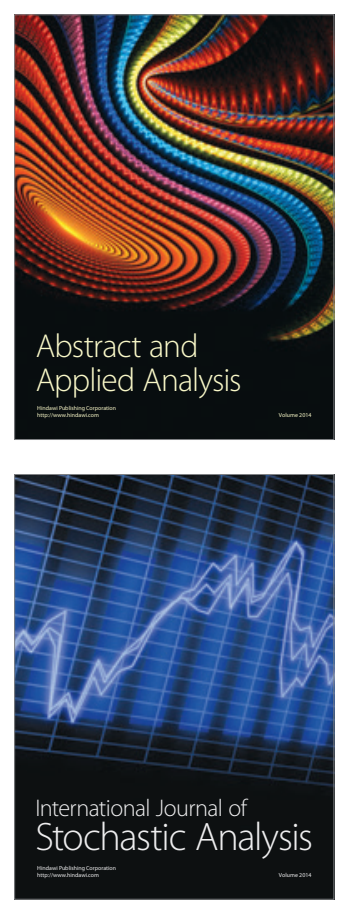

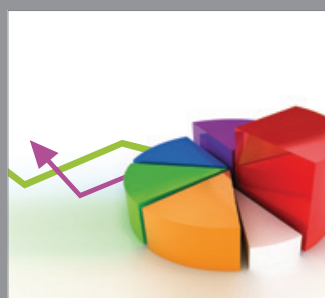

ournal of

Probability and Statistics

Promensencen
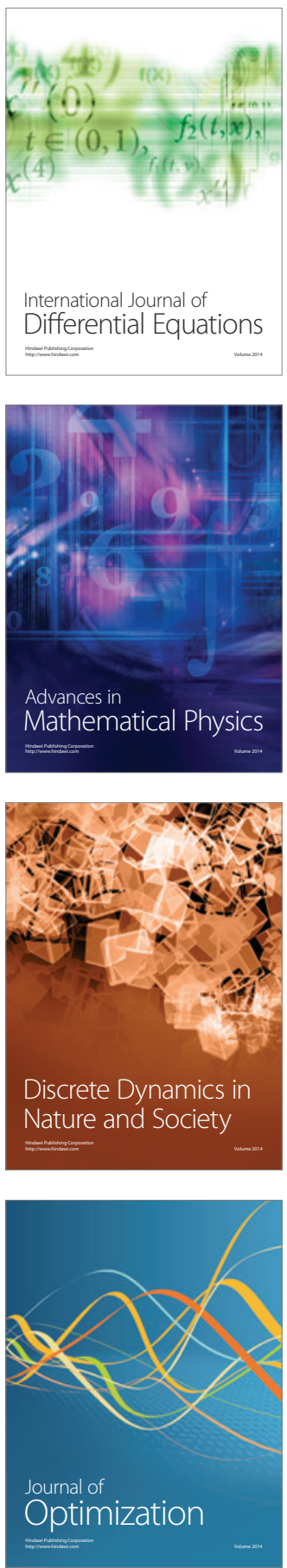